\title{
Raman Probe Based on Optically-Poled Double-Core Fiber
}

\author{
Brunetti, Anna Chiara; Margulis, Walter; Rottwitt, Karsten
}

Published in:

Imaging and Applied Optics Technical Digest

Publication date:

2012

Document Version

Publisher's PDF, also known as Version of record

Link back to DTU Orbit

Citation (APA):

Brunetti, A. C., Margulis, W., \& Rottwitt, K. (2012). Raman Probe Based on Optically-Poled Double-Core Fiber. In Imaging and Applied Optics Technical Digest (pp. STu1F.6). Optical Society of America.

\section{General rights}

Copyright and moral rights for the publications made accessible in the public portal are retained by the authors and/or other copyright owners and it is a condition of accessing publications that users recognise and abide by the legal requirements associated with these rights.

- Users may download and print one copy of any publication from the public portal for the purpose of private study or research.

- You may not further distribute the material or use it for any profit-making activity or commercial gain

- You may freely distribute the URL identifying the publication in the public portal

If you believe that this document breaches copyright please contact us providing details, and we will remove access to the work immediately and investigate your claim. 


\title{
Raman Probe Based on Optically-Poled Double-Core Fiber
}

\author{
Anna Chiara Brunetti ${ }^{1,2}$, Walter Margulis ${ }^{2}$, Karsten Rottwitt ${ }^{1}$ \\ 1. DTU Fotonik, Technical University of Denmark, Ørsteds Plads 343, DK-2800 Kgs. Lyngby, Denmark \\ 2. Acreo AB, Electrum 236, SE-164 40 Kista, Sweden \\ acbr@fotonik.dtu.dk
}

\begin{abstract}
A novel Raman probe based on an optically-poled double-core fiber is reported. Efficient in-fiber second-harmonic generation allows for Raman spectroscopy at $532 \mathrm{~nm}$ when illuminating the fiber with $1064 \mathrm{~nm}$ laser light. The concentrical cores of the fiber provide independent paths for the delivery of the excitation light and the collection of Raman scattering. Spectra of a sample of dimethyl sulfoxide acquired with this device are presented.

(C) 2012 Optical Society of America

OCIS codes: $300.6450,190.2620,060.2370$.
\end{abstract}

\section{Introduction}

Due to its ability to provide unambiguous and detailed molecular information on nearly any sample in a fast and non-destructive way, Raman spectroscopy represents an invaluable diagnostic and monitoring technique. In particular, when combined with fiber optics Raman spectroscopy can benefit from their flexibility and reduced size, therefore becoming the perfect candidate for remote in vivo analysis. Efforts to integrate in fiber the functions required for Raman analysis are thus motivated. The main challenges related to fiber-based Raman spectroscopy are the generation of the excitation light in the fiber and the efficient collection and filtering of the scattered light. In the case of singlefiber geometries the path separation between excitation and collection is also a major issue. While in-fiber Rayleighrejection filtering has previously been demonstrated [1,2], monolithic fiber lasers in the visible range are not so trivial to realize. Nevertheless, by optically poling the core of the fiber responsible for the delivery of the excitation light it is possible to achieve efficient second-harmonic generation (SHG) [3], thus being able to perform Raman spectroscopy at visible wavelengths despite having injected infrared (IR) light into the fiber. In-fiber SHG of up to $236 \mathrm{~mW}$ has been reported [4]. As for single-fiber geometries, coaxial double-core fibers provide an intrinsic separation between the excitation and collection paths (the inner and outer core, respectively), also allowing for a reduction of the fiber spectral background. Propagating the pump light in a small core allows for the illumination of the sample with high intensity, as required for nonlinear processes such as Raman scattering. Probes based on double-core fibers and doublecore fiber couplers for fluorescence and optical coherence tomography systems have previously been reported [5].

A complete all-in-fiber Raman spectroscopy system may be constructed by integrating a fiber laser, an opticallypoled double-core fiber, a double-core fiber coupler and an in-fiber Rayleigh-rejection filter. As an intermediate step towards this final goal, this work focuses on demonstrating that by optically poling the inner core of a double-core fiber it is possible to generate enough monochromatic visible light to perform Raman spectroscopy, and furthermore that the same waveguide is able to deliver the generated excitation light to a given sample through the inner core, and at the same time to collect the Raman scattering in its outer core.

\section{Materials and Method}

The double-core fiber used in this work, shown in Fig. 2.1 (a), is a step-index structure characterized by a central $7.5 \mu \mathrm{m}$ diameter germanium-doped inner core sorrounded by a $260 \mu \mathrm{m}$ pure silica outer core and by a fluorine-doped cladding of lower refractive index, for a total fiber diameter of $300 \mu \mathrm{m}$. For similar N.A. values, from simple geometrical considerations the collection in the outer core is 1200 times more efficient than in the inner core of the fiber.

\subsection{Optical Poling}

Prior to its employment in the Raman setup schematically illustrated in Fig. 2.1 (c), the double-core fiber was prepared for SHG by self-organization of a quasi-phase matched grating. The growth of the second harmonic (SH) light is known 
to be parametrically dependent on the amount of SH already present in the core [3], therefore it can be accelerated by briefly seeding the fiber with SH light, together with the fundamental IR [6]. For this reason, at first the $1064 \mathrm{~nm}$ light from a Q-switched mode-locked Nd:YAG laser (Quantronix) was frequency-doubled by means of a KTP crystal and coupled into the inner core of the fiber by means of a dichroic mirror designed to operate at $1064 \mathrm{~nm}$ and a 10x focusing lens (Nikon). After a few seconds of illumination with both green and IR light the nonlinear crystal was removed and the growth of the $\mathrm{SH}$ continued as a self-sustained process. Saturation occured after a few hours, with approx $0.83 \mathrm{~mW}$ average power of green light generated along the inner core of the fiber, out of the average $650 \mathrm{~mW}$ of IR light initially injected into it. It is worth noting that SHG happens in the first few centimeters of the waveguide, therefore in case the distal fiber tip gets damaged it is possible to re-cleave it without any change in the generated green light.
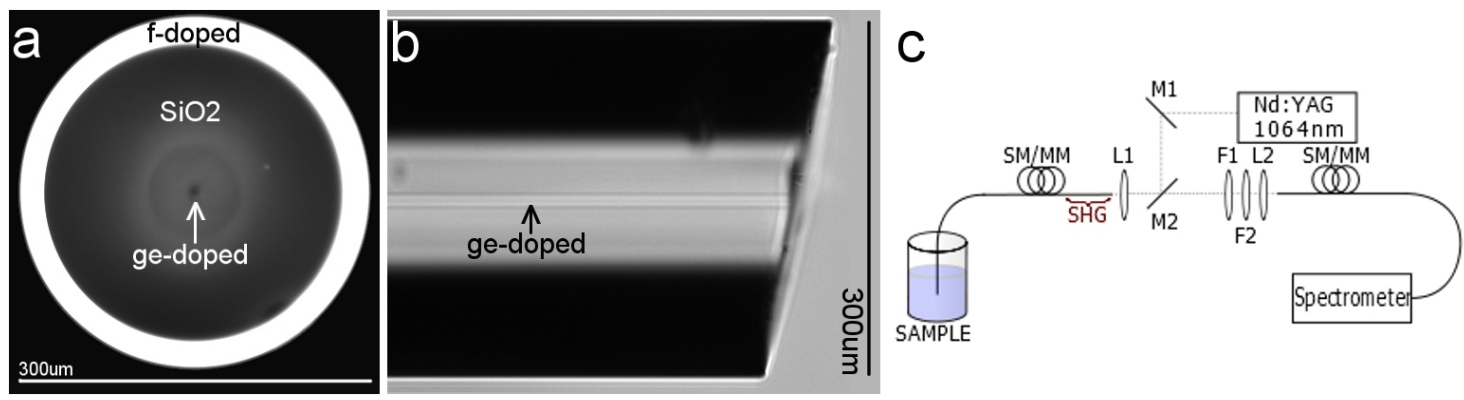

Fig. 1. (a) Cross-section of the dual-core fiber; (b) Angle-cleaved tip (about $11^{\circ}$ ) on the sample side; (c) Schematic illustration of the setup: M1,2 are mirrors (M2 is dichroic), L1,2 are 10x focusing lenses, F1 is a dual notch filter, F2 is a KG5 glass filter. The double-core fiber is indicated as $\mathrm{SM} / \mathrm{MM}$. The spectrometer is connected to a computer (not shown).

\subsection{Raman Spectroscopy}

The generated $532 \mathrm{~nm}$ light was used to perform Raman spectroscopy on a sample of Dimethyl Sulfoxide (DMSO, Merck), according to the setup shown in Fig. 2.1 (c). In particular, the sample was illuminated by the light exiting the inner core of the fiber, while the Raman-scattered light was collected by the outer core of the same waveguide. The fiber was inserted vertically into the sample, and a Raman spectrum was recorded. Subsequently, the fiber tip was extracted from the sample and held at a distance of about $10 \mathrm{~cm}$ from its surface, and a second spectrum was acquired to account for the interfering Raman signal generated in the fiber. Both spectra were acquired over a $15 \mathrm{~s}$ time, repeated twice to avoid artefacts. The fiber tip on the sample side had previously been cleaved at an angle of about $11^{\circ}$ by means of Vytran LDC400 fiber cleaver (see Fig. 2.1 (b)) to minimize reflections.

After propagating through the outer core of the fiber, the collected Raman signal was transmitted through the same dichroic mirror used to couple the IR laser light into the fiber, and was filtered by a dual 532 and $1064 \mathrm{~nm}$ notch filter (Edmund Optics), to eliminate the Rayleigh-scattered light and the IR light reflected by the other optical surfaces. A KG5 glass provided an additional filtering of the $1064 \mathrm{~nm}$ light. The collected Raman signal was then coupled into another piece of the same double-core fiber by a second 10x focusing lens (Nikon), and conveyed to a CCD-based spectrometer (QE65000, Ocean Optics), thermo-electrically cooled down to $-20^{\circ} \mathrm{C}$ to reduce dark current noise. The aperture slit of the spectrometer is $1 \mathrm{~mm}$ high and $50 \mu \mathrm{m}$ wide, therefore limiting the collection efficiency of the setup.

\section{Results and Discussion}

The generated $0.83 \mathrm{~mW}$ of $532 \mathrm{~nm}$ light proved to be intense enough to perform Raman spectroscopy on a sample of DMSO. Fig. 3 shows the recorded Raman spectrum, normalized to the spectrum acquired when the fiber tip was held outside the sample. Raman peaks at 1046, 1424, 2919 and $3005 \mathrm{~cm}^{-1}$, together with the double peak at 671 and $699 \mathrm{~cm}^{-1}$, are clearly identifiable. All the detected peaks match well the values reported in the literature [7].

The spectral normalization proved to be an easy and efficient way to extract the peaks located at low wavenumbers from the intense fiber spectral background, which would otherwise mask them, as shown in the inset of Fig. 3. This interfering signal is at least one order of magnitude more intense than the signal from DMSO, up to the point where it saturates the detector for wavenumbers smaller than $500 \mathrm{~cm}^{-1}$, even with acquisition times as short as $1 \mathrm{~s}$. For this reason, the spectral difference is only significant for wavenumbers larger than $500 \mathrm{~cm}^{-1}$. It is reasonable to think that 


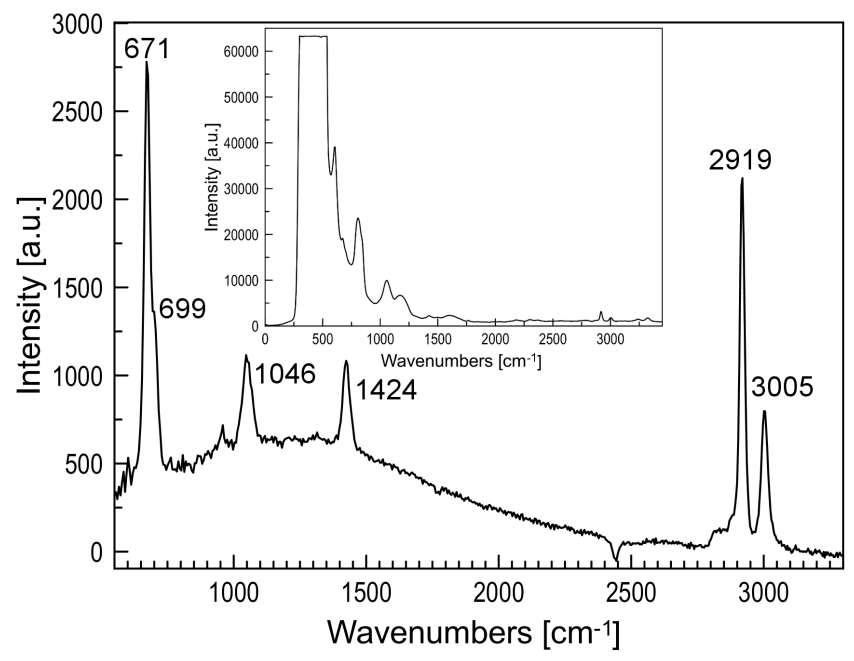

Fig. 2. Raman spectrum of DMSO acquired with the presented double-core fiber-based probe, normalized to the spectrum of the fiber. Inset: spectrum of DMSO as it appears before the normalization.

the strong fiber spectral background is related to that portion of the Raman background signal which is generated by the excitation light in the inner core but cannot be confined in it, due to its scattering angle, and therefore ends up being guided in the outer core. This was partially proved by the significant reduction of the Raman signal from the fiber achieved by angle-cleaving its tip. The notch appearing at about $2450 \mathrm{~cm}^{-1}$ is due to the spectral normalization.

\section{Conclusions}

Efficient SHG in a double-core fiber was demonstrated. The amount of generated green light proved to be enough to excite Raman scattering in sample of DMSO. The double-core structure allowed for the delivery of the generated excitation light to the sample through the inner core and the efficient collection of the scattered light in the outer core. The work described here represents a significant step towards an all-in-fiber Raman system in the visible range.

\section{Acknowledgments}

This work was financed by the Danish Council for Strategic Research, in the frame of the project "Towards robust fermentation processes by targeting population heterogeneity at microscale". Acreo Fiberlab is acknowledged for the special fiber fabrication.

\section{References}

1. M.J. Pelletier, "Fiber optic probe with integral optical filtering", USA Patent no.5862273 (1999).

2. A.C. Brunetti, L. Scolari, T. Lund-Hansen, J. Weirich and K. Rottwitt, "All-in-fiber Rayleigh-rejection filter for Raman spectroscopy", Electron. Lett., 48 (5), 275-276 (2012).

3. U. Österberg and W. Margulis, "Dye laser pumped by Nd:YAG laser pulses frequency doubled in a glass optical fiber", Opt. Lett. 11, 516-518 (1986).

4. A. Canagasabey, C. Corbari, A.V. Gladyshev, F. Liegeois, S. Guillemet, Y. Hernandez, M.V. Yashkov, A.Kosolapov, E.M. Dianov, M. Ibsen and P.G. Kazansky, "High-average-power second-harmonic generation from periodically poled silica fibers", Opt. Lett. 34, 2483-2485 (2009).

5. S.-Y. Ryu, H.-Y. Choi, M.-J. Ju, J.-H. Na, W.-J. Choi and B.-H. Lee, "The development of double clad fiber and double clad fiber coupler for fiber based biomedical imaging systems", J. Opt. Soc. Korea 13, 310-315 (2009).

6. R.H. Stolen and H.W.K. Tom, "Self-organized phase-matched harmonic generation in optical fibers", Opt. Lett. 12 (8), 585-587 (1987).

7. W.N. Martens, R.L. Frost, J. Kristof and J. Theo Kloprogge, "Raman spectroscopy of dimethyl sulphoxide and deuterated dimethyl sulphoxide at 298 and 77 K", J. Raman Spectrosc., 33, 84-91 (2002). 